\title{
Paclitaxel Chemotherapy Induces Long-Term Memory Impairment and Neuroinflammation in a Mouse Model of Breast Cancer Survivorship
}

\section{Ni-Chun Chung}

Monash Institute of Pharmaceutical Sciences

Aeson Chang

Monash Institute of Pharmaceutical Sciences

Ryan Gillis

Monash Institute of Pharmaceutical Sciences

\section{Erica Sloan}

Monash Institute of Pharmaceutical Sciences

Adam K Walker ( $\nabla$ a.walker@neura.edu.au )

Neuroscience Research Australia https://orcid.org/0000-0003-3772-5745

\section{Research Article}

Keywords: cancer surgery, memory impairment, chemotherapy, neuroinflammation, cancer survivorship

Posted Date: August 23rd, 2021

DOI: https://doi.org/10.21203/rs.3.rs-783452/v1

License: (c) (i) This work is licensed under a Creative Commons Attribution 4.0 International License. Read Full License 


\section{Abstract}

\section{Background}

Cancer-related cognitive impairment (CRCl) has been reported in cancer survivors 20 years or more after cancer treatment, and has been associated with sustained increases in circulating inflammatory biomarkers. One of the major risk factors for $\mathrm{CRCl}$ is chemotherapy, and preclinical studies typically examine the impact of chemotherapy in cancer naïve mice to evaluate potential mechanisms However, clinical evaluation of the long-term effects of chemotherapy cannot avoid the potential cumulative impact of preceding factors on the brain including the cancer itself and cancer surgery.

\section{Methods}

To evaluate the cumulative impact of cancer-related factors on cognitive impairment and hippocampal cytokine expression, we evaluated the effect of paclitaxel chemotherapy vs. placebo on a background of 67NR mammary carcinoma and surgical resection of the primary tumour in mice. Memory was assessed using the $Y$ maze test and novel object/novel place recognition test. Changes in hippocampal proinflammatory and anti-inflammatory cytokines, microglia and neuron markers were assessed using qRTPCR.

\section{Results}

Cancer and cancer surgery was sufficient to induce long-term memory impairment and sustained increases in hippocampal pro-inflammatory cytokines. Paclitaxel prolonged spatial memory impairment in the $\mathrm{Y}$ maze test and exacerbated hippocampal //6 and Tnfa mRNA expression compared with placebo treatment.

\section{Conclusions}

These findings suggest that cancer and cancer surgery can sensitise the brain to an exaggerated neuroinflammatory response to chemotherapy, and may contribute to sustained chemotherapy-induced cognitive impairment observed in cancer survivors.

\section{Background}

As the survival rate of cancer improves, the long-term impact of cancer and cancer treatment on breast cancer survivors becomes a significant public health concern. After cessation of cancer treatment, up to $50 \%$ of breast cancer survivors have long-term cognitive impairment in verbal short-term memory, attention, concentration, psychomotor function, information processing speed, or executive function (15). These cognitive deficits have been associated with altered brain activity and structural damage to brain areas including white matter tracts, and reduced hippocampal volume $(2,3,6-8)$, and with changes in systemic and brain glucose metabolism (9-12). 
Treatments including chemotherapy, hormonal therapy and radiation are well known to contribute to these cognitive problems. It is now also accepted that factors that precede adjuvant treatment - such as the cancer itself and cancer surgery -may also contribute to cognitive impairment in cancer patients by inducing inflammation in the body and brain $(4,13-15)$. While clinical evaluation of the long-term effects of chemotherapy cannot avoid the potential cumulative impact of these preceding factors on the brain, most preclinical studies to date have investigated the impact of chemotherapy on the brain and behaviour in the absence of other factors $(16,17)$. That approach enables greater precision in isolating the mechanisms of cognitive impairment that occur from chemotherapy. The resulting studies in nontumour bearing mice have demonstrated that chemotherapy enhances (neuro)inflammation and, neural damage, reduces neurogenesis, activates physiological stress pathways, and contributes to cognitive impairment and behavioural changes (18-20). However, evaluating the effect of chemotherapy on cognitive impairment in isolation ignores the contribution of cancer and other treatments such as surgery, which may plausibly compound the effects of adjuvants therapies like chemotherapy on the brain. The contention that multiple events during the cancer journey can induce a synergistic or cumulative impact on the brain is supported by Winocur et al. who demonstrated worse memory performance in cancerbearing mice treated with chemotherapy (methotrexate and 5-fluorouracil) compared to either cancer or chemotherapy alone (21). The sustained impact of chemotherapy in cancer free survivors was not evaluated in that study due to the aggressive nature of the metastatic transgenic model of breast cancer (MMTVneu FVB) used [19]. Therefore, to address the long-term impact of paclitaxel chemotherapy on memory in a clinically relevant setting, here we used a less aggressive murine model of mammary carcinoma, and evaluated the effect of adjuvant chemotherapy after primary tumour resection.

\section{Materials And Methods}

\section{Animals}

BALB/c female mice (6-8 weeks old) (Monash University, Australia) were single caged in a temperature and humidity-controlled environment with a 12/12-h reverse dark-light cycle (lights on at 19:00). Food and water were available ad libitum. All procedures involving mice were carried out under protocols approved by the Monash University Animal Ethics Committee (protocol number MIPS2015.01 and 2015.16). Animals were monitored daily for distress. Humane endpoints based on body weight loss, body condition scoring and metastatic progression were in place. Mice were euthanized with $\mathrm{CO}_{2}$.

\section{Breast cancer model}

To generate syngenic mammary tumours, $1 \times 10^{6} 67$ NR cells in $20 \mu \mathrm{L}$ PBS were injected into the 4th left mammary gland of anaesthetized BALB/c mice. Once primary tumours were palpable, tumour growth was monitored by caliper measurement and tumour volume determined using the following formula: (length $\mathrm{x}$ width $\left.{ }^{2}\right) / 2$. Cell culture details are provided in Supplementary Material.

\section{Breast cancer surgery}


The primary tumour, left inguinal mammary fatpad and adjacent left inguinal lymph node were resected under sterile conditions and $3 \%$ isoflurane. The wound site was then sutured. Buprenorphine $(1 \mu \mathrm{g} / 100$ $\mu \mathrm{L}$, s.c.) was administered prior to surgery and every $12 \mathrm{~h}$ after surgery for 3 doses in total. All mice underwent the same anesthesia procedure and received buprenorphine. Body weight and burrowing activity and body condition scoring were recorded daily to assess sickness behaviour and activity in response to surgery.

\section{Paclitaxel treatment}

Mice were randomly allocated to placebo or paclitaxel treatment groups while ensuring equal distribution of mice to each group based on performance in a NOPR and $\mathrm{Y}$ maze test after tumour cell injection and primary tumour burden (Fig S1A-C). Mice were injected intraperitoneally with $10 \mathrm{mg} / \mathrm{kg}$ paclitaxel (Assay Matrix and Sigma-Aldrich, USA) solubilized in vehicle (Cremorphor EL:ethanol:saline, 1:1:10) or an equal volume of placebo every second day for 2 weeks as in (22). Within the 13 mice of the placebo group, to confirm there was no side effect of Cremorphor EL on behaviour (23), 8 mice were administered saline and the other 5 mice were administered vehicle (Cremorphor EL:ethanol:saline, 1:1:10). No significant differences were observed between saline and vehicle treated mice for any measure, therefore these were collapsed and treated as a single placebo group.

\section{Memory and sickness behaviour assays}

Attention and episodic memory were tested using a novel object/novel place recognition (NOPR) test as previously described (22). Spatial memory was tested using the $Y$ maze. Behaviour was video recorded and analyzed using tracking software (Viewer ${ }^{3}$, Biobserve, Germany). Locomotor activity was assessed as total movement $(\mathrm{cm})$ in both tests. Daily body weights and home cage burrowing activity of mice were recorded to assess sickness behaviour and activity. Details are available in Supplementary Material.

\section{RNA extraction and quantitative reverse transcriptase polymerase chain reaction (qRT-PCR)}

To explore the association between inflammation in our cancer-free survivor mice with the sustained changes in hippocampal-dependent memory, we assessed neuroinflammation and neuronal activityrelated gene expression in the hippocampus of paclitaxel and placebo treated mice, untreated 8-week-old (pre-cancer baseline age), and untreated age-matched 19-week-old control groups (baseline for experimental end point). All the groups were normalized to the 8-week-old control group for general comparison. qRT-PCR was used to assess gene expression changes in 100 ng RNA with iScript ${ }^{\text {TM }}$ One-Step RT-PCR Kit for Probes (Bio-Rad, USA). Taqman probes targeted Actb, $/ 11 b,\|/ 6\| 10$, Tnfa, Iba1/Aif1, Bdnf, and $R b f o x 3 / N e u N$. The RNA and probe mix was subjected to 40 PCR amplification cycles. Each sample was run in triplicate and the genes of interest were normalized to the housekeeper gene $\beta$-actin $(A c t b)$ via the $2^{-\Delta \Delta C t}$ method. Details provided in Supplementary Material.

\section{Statistical analysis}


Paired student's t-tests were used to assess tumour-induced memory performance from baseline in the $Y$ Maze and NOPR test. Repeated two-way analyses of variance (ANOVAs) were used to analyze behaviour in the NOPR test and Y maze, locomotor activity, burrowing activity, and body weight for paclitaxel- vs placebo-treated groups. Unpaired Student's t-test or between subjects one-way ANOVAs were used to analyze data from cytokine expression assays and spleen mass. For within group planned comparisons between baseline and other time points, Dunnett's multiple comparison test was used. For between group planned comparisons independent $t$-tests were used with a adjusted based on number of comparisons. Statistical significance was set at a less than or equal to 0.05 .

\section{Results}

\section{Paclitaxel exacerbates cancer- and cancer surgery-induced Tnfa and //6 gene expression in the hippocampus}

To investigate the effect of cancer and surgery on the brain, mice with 67NR tumours underwent surgical resection and we investigated the long-term effect on cytokine expression in the brain (Fig. 1A). 58 days after tumour resection, mice that previously had tumours showed a significant increase in expression of hippocampal pro-inflammatory cytokines including $/ / 1 b\left(F_{(3,26)}=16.15, p<0.0001\right)$, $\operatorname{Tnfa}\left(F_{(3,26)}=66.70, p\right.$ $<0.0001)$, II6 $\left(F_{(3,26)}=85.31, p<0.0001\right)$, and anti-inflammatory $\| 10\left(F_{(3,26)}=7.29, p<0.001\right)$ compared to cancer naïve mice. Paclitaxel treatment after tumour resection significantly exacerbated expression of Tnfa and II6 ( $p<0.05$ for both) (Fig. 1B). To determine if increases in hippocampal cytokines were associated with changes in hippocampal resident microglia/macrophages or changes in the neuron population, we assessed expression of Iba $1, R f o x 3$ (NeuN) and brain derived nerve growth factor (Bdnf). Mice that had previously had tumours resected had increased Iba1 expression, suggesting elevated numbers or activation of brain microglia/macrophages $\left(F_{(3,26)}=47.40, p<0.0001\right)$ (Fig. 1C). Rfox3 $(\mathrm{NeuM})$ is a gene expressed in neural tissue and was significantly increased in mice that previously had tumours compared to age-mated untreated controls $\left(F_{(3,26)}=42.32, p<0.0001\right)$ such that they had equivalent expression to 8 week old untreated control mice $(p>0.05)$ (Fig. 1D). Increased hippocampal $B d n f$ mRNA expression was also observed $\left(F_{(3,26)}=47.40, p<0.0001\right)$ (Fig. 1E), which could be an early response to behaviour tests that was performed $24 \mathrm{~h}$ prior to sample collection and is consistent with previous studies (24). Paclitaxel did not significantly impact Iba1, Rfox3 or Bdnf.

To confirm that these changes were not due to aging given the length of the experiment, we assessed hippocampal gene expression for all genes in 8 week old and 19 week old untreated mice coinciding with the ages of mice at the start and end of the experiment. No age-related changes hippocampal mRNA expression was observed for cytokine, Iba 1 or bdnf expression. Older mice showed a decrease in Rfox 3 $(\mathrm{NeuN})\left(F_{(3,26)}=42.32, p<0.0001\right)$, which has been reported for the protein upon aging $(25)$.

To determine if hippocampal inflammation was associated with peripheral inflammation, we assessed spleen weight - a measure of myeloid expansion and inflammation in 67NR tumour bearing mice (26- 
29). No significant differences were observed in relation to spleen weight by 37 days after treatment with paclitaxel (58 days after 67NR primary tumour resection) (Fig. 1F).

\section{Cancer-related memory impairment is and prolonged by paclitaxel treatment}

We examined whether paclitaxel exacerbated or prolonged memory impairment in mice that previously had cancer. Consistent with previous findings (28), prior to resection mice with tumours showed memory impairment in the Y-maze test $\left(\mathrm{t}_{(26)}=3.28, \mathrm{p}<0.01\right)$ and NOPR test $\left(t_{(26)}=3.60, p<0.01\right)$ (Fig S1A and B). To assess the impact of treatment with paclitaxel on memory, mice were tested in the NOPR test and $Y$ maze for 6 weeks starting $24 \mathrm{~h}$ after the end of paclitaxel or placebo treatment. Performance in the $Y$ maze test changed over time $\left(F_{(3.774,87.56)}=3.31, p=0.016\right)(F i g .2 A)$. Multiple comparisons revealed that memory was significantly worse than pre-cancer memory only 2 days after treatment for mice placebotreated mice $(p<0.05)$. However, memory was significantly worse than pre-cancer memory at $48 \mathrm{~h}(p<$ $0.05) 9$ days $(p<0.01)$ and 37 days $(p<0.05)$ after cessation of treatment with paclitaxel. Paclitaxeltreated mice performed worse than placebo-treated controls at each time point following baseline, which reached significance at 37 days after treatment cessation $\left(t_{(23)}=2.60, p=0.05\right)$ (Fig. 2A). Performance in the in the NOPR test also changed over time $\left(F_{(4.90,118.4)}=4.63, p=0.00007\right)$ (Fig. 2B). Memory was worse compared to the pre-cancer baseline at all time points regardless of paclitaxel or placebo treatment $(p<0.001)$. Paclitaxel did not significantly affect memory in the NOPR test compared to placebo-treated mice $(p>0.05)$ (Fig. 2B).

To confirm that memory impairment did not reflect sickness, cancer-induced fatigue or inactivity, we assessed sickness responses and burrowing activity. While surgery induced a temporary reduction in body weight due to both the removal of the tumour mass and a transient impact of surgery, all mice increased in body weight over the course of the experiment and there were no differences between mice treated with paclitaxel versus placebo (Fig. 3A). Paclitaxel did not significantly affect locomotor activity in the $Y$ maze or NOPR test (Fig. 3B and C). Burrowing activity declined from baseline until the end of the experiment $\left(F_{(5.08,144.4)}=4.48, p=0.0004\right)$. Treatment with paclitaxel also significantly affected burrowing activity, vs. placebo $\left(F_{(1,29)}=9.62, p=0.004\right)$. Planned comparisons revealed that the placebo treated mice burrowed significantly less than paclitaxel treated mice in response to tumours and between day 8 and day 29 after chemotherapy or placebo treatment (all $p<0.05)$ (Fig. 3D).

\section{Discussion}

Paclitaxel is a chemotherapeutic agent that is commonly used to treat diverse solid tumours and has been associated with cognitive decline (30). However, the effect of paclitaxel in the context of cancer and cancer resection surgery has not been described. To determine if there is a cumulative long-term impact on cognition, we developed a mouse model of cancer survivorship to evaluate long-term memory in mice after the resection of a cancer and treatment with adjuvant chemotherapy. Evaluation of the model 
demonstrated a sustained impact of cancer, surgery and adjuvant paclitaxel on the brain. 37 days after cessation of chemotherapy, mice showed memory impairment. This was found in tests that examine spatial memory (Y Maze, NOPR) and recognition memory (NOPR). Previous studies by us and others show that memory and inflammation is impacted by each of these single events: having a cancer $(21,28$, $31-34)$, cancer surgery $(35,36)$, and treatment with paclitaxel $(18-20,22)$. The findings of the current study extend these observations by demonstrating a cumulative effect of cancer and chemotherapy on memory and brain inflammation that results in long-term impairment.

Using this model of paclitaxel chemotherapy in cancer naïve mice, we previously observed no change in brain cytokine mRNA or protein (22). The findings here indicate that cancer and surgery sensitise the brain to chemotherapy-induced neuroinflammation, which persists long after chemotherapy treatment has ended. The discovery that paclitaxel exacerbated Tnfa and $I / 6$ mRNA expression in the hippocampus, supports the contention of a synergistic effect of cancer and chemotherapy on brain inflammation. These findings are supported by previous research. Consistent with this, using a mouse model of mammary carcinoma, Pyter et al. (2014) demonstrated that peripheral tumours can prime or sensitise the brain to inflammation which resulted in an exacerbated inflammatory response to lipopolysaccharide (32). Here, we confirm the capacity for tumours and cancer surgery to prime the brain and extend these findings to a clinically relevant context by demonstrating that a peripheral tumour and cancer surgery can prime the brain for an exaggerated neuroinflammatory response to chemotherapeutic agents such as paclitaxel long after the tumour has been resected. To control for the impact of tumour burden on inflammation, in this study we ensured uniform tumour sizes between paclitaxel and placebo treatment groups, and resected all tumours at the same time. Future studies should examine whether cancer stage or tumour size influences the magnitude of brain sensitization to chemotherapy.

The findings suggest that brain-resident macrophages may contribute to neural inflammation in longterm cancer survivor mice. While paclitaxel specifically exacerbated Tnfa and $/ 16 \mathrm{mRNA}$, all cytokines assessed were elevated in mice that previously had tumours, and this was sustained 58 days after tumour resection compared with cancer-naïve mice. Iba 1 was similarly increased following cancer and cancer treatment, suggesting increased proliferation or activation of macrophages in the hippocampus consistent with findings demonstrating a synergistic effect of mastectomy and cancer in another preclinical breast cancer model (35). Spleen weights did not differ by experimental endpoint suggesting that peripheral myeloid cell expansion and inflammation caused by 67NR tumours may have subsided following primary tumour resection. Comparison between 19 week and 8 week old cancer naïve mice indicated that age did not contribute to neuroinflammation. Taken together, these findings suggest that brain resident macrophages remain activated and may drive sustained elevations in hippocampal cytokines thus creating a new "baseline" level of brain cytokines.

In future studies it will be important to understand the role of hippocampal TNF $a$ and IL6 in cancerrelated $\mathrm{Cl}$ (Fig. 1). Both TNF-a and IL-6 or their receptors have been reported to still be elevated in the blood of breast cancer survivors 1-12 years after chemotherapy, and are associated with reduced hippocampal volume and cognitive impairment (2). Those clinical findings suggest that the sustained 
increase in circulating TNF- $a$ and IL- 6 in breast cancer survivors may serve as biomarkers for elevated hippocampal inflammation long after cancer treatment. TNF- $a$ and IL-6 play important roles in hippocampal-dependent memory by impeding long-term potentiation (LTP). IL- 6 can inhibit LTP by reducing MAPK/ERK through prolonged activation of STAT3 $(37,38)$. High levels of TNF-a can affect neural transmission by causing an imbalance between excitatory and inhibitory transmission by enhancing PI3K-dependent AMPA trafficking to increase $\mathrm{Ca}^{2+}$ influx and decreasing incorporation of $G_{A B A}$ receptor to reduce inhibitory transmission. This can lead to apoptosis of hippocampal neurons $(20,38-40)$. In the current study neither of the neuronal and neurogenesis related genes Rfox 3 or Bdnf were reduced in paclitaxel treated mice, suggesting that the elevations in Tnfa and $/ 16$ may not be severe enough to cause long-term neuronal death. However, future studies should confirm these mRNA findings using protein homogenate and immunohistochemistry techniques. Nevertheless, these findings support the evaluation of TNF-a and IL-6 neutralizing antibodies as potential therapeutics for cancer survivors who suffer paclitaxel chemotherapy-induced cognitive impairment. In support of this contention, treatment with the TNF-a inhibitor thalidomide during paclitaxel treatment has been shown to prevent spatial memory impairment and neuron apoptosis in the hippocampus (20).

In contrast to clinical studies, which use cognitive function after cancer diagnosis or cancer surgery as the baseline to determine dysfunction induced by chemotherapy, here, we compared changes to a true pre-cancer baseline, in addition to between-subjects analysis of paclitaxel treated mice with placebotreated controls. Paclitaxel treatment prolonged spatial memory impairment measured by the $Y$ maze test whereas placebo treated mice eventually returned to baseline function. However, regardless of chemotherapy treatment, cancer and cancer treatment caused memory impairment in the $Y$ maze test for several weeks and impairment in the NOPR test was sustained in both paclitaxel and placebo treated mice. In conjunction with the widespread hippocampal inflammatory response caused by tumours, these memory findings suggest that cancer and cancer surgery contribute to the profound long-term changes in brain function in addition to chemotherapy. However, the findings of this study may be confounded by additional risk factors including 3 months of ageing across the course of the experiment, and the stress of multiple injections. For instance, studies have shown that prior stresses amplify subsequent inflammatory responses: mice exhibited higher inflammatory responses to stress (induced by injection of saline or tail suspension) 14 days after primary tumour resection compared with their control groups (35, 36). Nevertheless, the findings of this study suggest that longitudinal clinical studies that assess cognitive changes in cancer patients or survivors after adjuvant chemotherapy compared to a prechemotherapy baseline, may fail to observe quantifiable cognitive decline relative to cognition at cancer diagnosis. This bias may lead some studies to underestimate or underdiagnose cognitive impairment in cancer survivors after adjuvant chemotherapy.

\section{Conclusion}

This study demonstrates long-term cognitive impairment and a cumulative impact of cancer treatment and the cancer itself on the brain in a mouse model of cancer survivorship. For the first time, we 
demonstrate that tumour-induced neuroinflammation and memory impairment persists for months in mice, even after tumours are resected, and that chemotherapy can exacerbate tumour and surgeryinduced neuroinflammation and prolong memory impairment.

\section{Abbreviations}




\begin{tabular}{|c|c|}
\hline Actb & Actin Beta \\
\hline Aif1 & Allograft inflammatory factor 1 \\
\hline AMPA & a-Amino-3-hydroxy-5-methyl-4-isoxazolepropionic acid \\
\hline ANOVA & Analysis of variance \\
\hline Bdnf & Brain-derived neurotrophic factor \\
\hline BW & Body weight \\
\hline $\mathrm{Ca}^{2+}$ & Calcium ion \\
\hline $\mathrm{CO}_{2}$ & Carbon dioxide \\
\hline $\mathrm{CRCl}$ & Cancer-related cognitive impairment \\
\hline $\mathrm{Ct}$ & Cycle threshold \\
\hline Ctx & Chemotherapy \\
\hline ERK & Extracellular signal-regulated kinase \\
\hline $\mathrm{GABA}_{\mathrm{A}}$ receptor & Y-Aminobutyric acid type A receptor \\
\hline lba1 & lonized calcium binding adaptor molecule 1 \\
\hline$\| 1 \mathrm{~b}$ & Interleukin 1 beta \\
\hline 116 & Interleukin 6 \\
\hline$\| 10$ & Interleukin 10 \\
\hline LTP & Long-term potentiation \\
\hline MAPK & Mitogen-activated protein kinase \\
\hline mRNA & Messenger ribonucleic acid \\
\hline NeuN & Neuronal nuclei \\
\hline NOPR & Naval objective/novel place recognition \\
\hline $\mathrm{PI} 3 \mathrm{~K}$ & Phosphoinositide 3-kinase \\
\hline PBS & Phosphate-buffered saline \\
\hline qRT-PCR & Quantitative reverse transcriptase polymerase chain reaction \\
\hline Rbfox3 & RNA binding Fox-1 homolog 3 \\
\hline$S$ & Surgery \\
\hline STAT3 & Signal transducer and activator of transcription 3 \\
\hline
\end{tabular}




\begin{tabular}{|ll|}
\hline Actb & Actin Beta \\
\hline T & Tumour \\
\hline Tnfa & Tumor necrosis factor alpha \\
\hline
\end{tabular}

\section{Declarations}

\section{Ethics approval and consent to participate}

All procedures involving mice were carried out under protocols approved by the Monash University Animal Ethics Committee (protocol number MIPS2015.01 and 2015.16).

\section{Consent for publication}

Not applicable.

\section{Availability of data and materials}

The datasets used and/or analysed during the current study are available from the corresponding author on reasonable request.

\section{Competing interests}

The authors declare that they have no competing interests.

\section{Funding}

This work was supported by the National Breast Cancer Foundation, Australia [PF-15-014, IIRS-20-025] and the National Health and Medical Research Council [1147498]. AKW is supported by the Schizophrenia Research Institute, Australia and Neuroscience Research Australia.

\section{Authors' contributions}

NC, EKS and AKW were involved in all aspects of this study including conception, design, data acquisition, analysis, interpretation, and manuscript preparation. AC was involved in data acquisition, interpretation, and manuscript preparation. RDG was involved in data acquisition and manuscript preparation. All authors have approved this submission and take responsibility for the accuracy and integrity of the manuscript.

\section{Acknowledgements}

Not applicable.

\section{References}


1. Ahles TA, Saykin AJ. Breast cancer chemotherapy-related cognitive dysfunction. Clin Breast Cancer. 2002;3 Suppl 3:S84-90.

2. Kesler S, Janelsins M, Koovakkattu D, Palesh O, Mustian K, Morrow G, Dhabhar FS. Reduced hippocampal volume and verbal memory performance associated with interleukin- 6 and tumor necrosis factor-alpha levels in chemotherapy-treated breast cancer survivors. Brain Behav Immun. 2013;30 Suppl:S109-16.

3. Kesler SR, Kent JS, O'Hara R. Prefrontal cortex and executive function impairments in primary breast cancer. Arch Neurol. 2011;68(11):1447-53.

4. Janelsins MC, Heckler CE, Peppone LJ, Ahles TA, Mohile SG, Mustian KM, Palesh O, O'Mara AM, Minasian LM, Williams AM, Magnuson A, Geer J, Dakhil SR, Hopkins JO, Morrow GR. Longitudinal Trajectory and Characterization of Cancer-Related Cognitive Impairment in a Nationwide Cohort Study. J Clin Oncol. 2018:JCO2018786624.

5. Joly F, Lange M, Dos Santos M, Vaz-Luis I, Di Meglio A. Long-Term Fatigue and Cognitive Disorders in Breast Cancer Survivors. Cancers (Basel). 2019;11(12).

6. McDonald BC, Conroy SK, Smith DJ, West JD, Saykin AJ. Frontal gray matter reduction after breast cancer chemotherapy and association with executive symptoms: a replication and extension study. Brain Behav Immun. 2013;30 Suppl:S117-25.

7. Inagaki M, Yoshikawa E, Matsuoka Y, Sugawara Y, Nakano T, Akechi T, Wada N, Imoto S, Murakami K, Uchitomi Y. Smaller regional volumes of brain gray and white matter demonstrated in breast cancer survivors exposed to adjuvant chemotherapy. Cancer. 2007;109(1):146-56.

8. Matsos A, Loomes M, Zhou I, Macmillan E, Sabel I, Rotziokos E, Beckwith W, Johnston IN. Chemotherapy-induced cognitive impairments: White matter pathologies. Cancer Treat Rev. 2017;61:6-14.

9. Krak NC, van der Hoeven JJ, Hoekstra OS, Twisk JW, van der Wall E, Lammertsma AA. Measuring [(18)F]FDG uptake in breast cancer during chemotherapy: comparison of analytical methods. Eur J Nucl Med Mol Imaging. 2003;30(5):674-81.

10. Mankoff DA, Dunnwald LK, Gralow JR, Ellis GK, Schubert EK, Tseng J, Lawton TJ, Linden HM, Livingston RB. Changes in blood flow and metabolism in locally advanced breast cancer treated with neoadjuvant chemotherapy. J Nucl Med. 2003;44(11):1806-14.

11. Pomykala KL, Ganz PA, Bower JE, Kwan L, Castellon SA, Mallam S, Cheng I, Ahn R, Breen EC, Irwin $\mathrm{MR}$, Silverman $\mathrm{DH}$. The association between pro-inflammatory cytokines, regional cerebral metabolism, and cognitive complaints following adjuvant chemotherapy for breast cancer. Brain Imaging Behav. 2013;7(4):511-23.

12. Silverman DH, Dy CJ, Castellon SA, Lai J, Pio BS, Abraham L, Waddell K, Petersen L, Phelps ME, Ganz PA. Altered frontocortical, cerebellar, and basal ganglia activity in adjuvant-treated breast cancer survivors 5-10 years after chemotherapy. Breast Cancer Res Treat. 2007;103(3):303-11.

13. Ahles TA, Saykin AJ, McDonald BC, Furstenberg CT, Cole BF, Hanscom BS, Mulrooney TJ, Schwartz GN, Kaufman PA. Cognitive function in breast cancer patients prior to adjuvant treatment. Breast 
cancer research and treatment. 2008;110(1):143-52.

14. Hedayati E, Schedin A, Nyman H, Alinaghizadeh H, Albertsson M. The effects of breast cancer diagnosis and surgery on cognitive functions. Acta Oncol. 2011;50(7):1027-36.

15. Wefel JS, Lenzi R, Theriault R, Buzdar AU, Cruickshank S, Meyers CA. 'Chemobrain' in breast carcinoma?: a prologue. Cancer. 2004;101(3):466-75.

16. Vardy JL, Dhillon HM, Pond GR, Rourke SB, Bekele T, Renton C, Dodd A, Zhang H, Beale P, Clarke S, Tannock IF. Cognitive Function in Patients With Colorectal Cancer Who Do and Do Not Receive Chemotherapy: A Prospective, Longitudinal, Controlled Study. J Clin Oncol. 2015;33(34):4085-92.

17. Wefel JS, Saleeba AK, Buzdar AU, Meyers CA. Acute and late onset cognitive dysfunction associated with chemotherapy in women with breast cancer. Cancer. 2010;116(14):3348-56.

18. Huehnchen P, Boehmerle W, Springer A, Freyer D, Endres M. A novel preventive therapy for paclitaxelinduced cognitive deficits: preclinical evidence from C57BL/6 mice. Translational psychiatry. 2017;7(8):e1185.

19. Lee BE, Choi BY, Hong DK, Kim JH, Lee SH, Kho AR, Kim H, Choi HC, Suh SW. The cancer chemotherapeutic agent paclitaxel (Taxol) reduces hippocampal neurogenesis via down-regulation of vesicular zinc. Sci Rep. 2017;7(1):11667.

20. Li Z, Zhao S, Zhang HL, Liu P, Liu FF, Guo YX, Wang XL. Proinflammatory Factors Mediate PaclitaxelInduced Impairment of Learning and Memory. Mediators Inflamm. 2018;2018:3941840.

21. Winocur G, Berman H, Nguyen M, Binns MA, Henkelman M, van Eede M, Piquette-Miller M, Sekeres MJ, Wojtowicz JM, Yu J, Zhang H, Tannock IF. Neurobiological Mechanisms of Chemotherapyinduced Cognitive Impairment in a Transgenic Model of Breast Cancer. Neuroscience. 2018;369:5165.

22. Chang A, Chung NC, Lawther AJ, Ziegler Al, Shackleford DM, Sloan EK, Walker AK. The AntiInflammatory Drug Aspirin Does Not Protect Against Chemotherapy-Induced Memory Impairment by Paclitaxel in Mice. Frontiers in oncology. 2020;10:564965.

23. Gelderblom H, Verweij J, Nooter K, Sparreboom A. Cremophor EL: the drawbacks and advantages of vehicle selection for drug formulation. Eur J Cancer. 2001;37(13):1590-8.

24. Mizuno M, Yamada K, Olariu A, Nawa H, Nabeshima T. Involvement of Brain-Derived Neurotrophic Factor in Spatial Memory Formation and Maintenance in a Radial Arm Maze Test in Rats. The Journal of Neuroscience. 2000;20(18):7116.

25. Soreq L, Consortium UKBE, North American Brain Expression C, Rose J, Soreq E, Hardy J, Trabzuni D, Cookson MR, Smith C, Ryten M, Patani R, Ule J. Major Shifts in Glial Regional Identity Are a Transcriptional Hallmark of Human Brain Aging. Cell Rep. 2017;18(2):557-70.

26. Bronte V, Pittet MJ. The spleen in local and systemic regulation of immunity. Immunity. 2013;39(5):806-18.

27. Steenbrugge J, Vander Elst N, Demeyere K, De Wever O, Sanders NN, Van Den Broeck W, Dirix L, Van Laere S, Meyer E. Comparative Profiling of Metastatic 4T1- vs. Non-metastatic Py230-Based 
Mammary Tumors in an Intraductal Model for Triple-Negative Breast Cancer. Front Immunol. 2019;10:2928.

28. Walker AK, Chang A, Ziegler Al, Dhillon HM, Vardy JL, Sloan EK. Low dose aspirin blocks breast cancer-induced cognitive impairment in mice. PLoS One. 2018;13(12):e0208593.

29. Walker II WH, Borniger JC, Surbhi, Zalenski AA, Muscarella SL, Fitzgerald JA, Zhang N, Gaudier-Diaz MM, DeVries AC. Mammary Tumors Induce Central Pro-inflammatory Cytokine Expression, but Not Behavioral Deficits in Balb/C Mice. Sci Rep. 2017;7(1):8152.

30. da Costa R, Passos GF, Quintao NLM, Fernandes ES, Maia J, Campos MM, Calixto JB. Taxaneinduced neurotoxicity: Pathophysiology and therapeutic perspectives. Br J Pharmacol. 2020;177(14):3127-46.

31. Pyter LM, Cochrane SF, Ouwenga RL, Patel PN, Pineros V, Prendergast BJ. Mammary tumors induce select cognitive impairments. Brain Behav Immun. 2010;24(6):903-7.

32. Pyter LM, El Mouatassim Bih S, Sattar H, Prendergast BJ. Peripheral tumors alter neuroinflammatory responses to lipopolysaccharide in female rats. Brain Res. 2014;1552:55-63.

33. Yang M, Kim J, Kim JS, Kim SH, Kim JC, Kang MJ, Jung U, Shin T, Wang H, Moon C. Hippocampal dysfunctions in tumor-bearing mice. Brain Behav Immun. 2014;36:147-55.

34. Vichaya EG, Vermeer DW, Christian DL, Molkentine JM, Mason KA, Lee JH, Dantzer R. Neuroimmune mechanisms of behavioral alterations in a syngeneic murine model of human papilloma virusrelated head and neck cancer. Psychoneuroendocrinology. 2017;79:59-66.

35. Emmer KM, Walker WH, 2nd, Zhang N, DeVries AC. Mammary tumor and mastectomy synergistically promote neuroinflammation in a breast cancer survivor model. Brain Res. 2019;1707:133-40.

36. Pyter LM, Suarez-Kelly LP, Carson WE, 3rd, Kaur J, Bellisario J, Bever SR. Novel rodent model of breast cancer survival with persistent anxiety-like behavior and inflammation. Behav Brain Res. 2017;330:108-17.

37. Tancredi V, D'Antuono M, Cafe C, Giovedi S, Bue MC, D'Arcangelo G, Onofri F, Benfenati F. The inhibitory effects of interleukin- 6 on synaptic plasticity in the rat hippocampus are associated with an inhibition of mitogen-activated protein kinase ERK. J Neurochem. 2000;75(2):634-43.

38. Donzis EJ, Tronson NC. Modulation of learning and memory by cytokines: signaling mechanisms and long term consequences. Neurobiol Learn Mem. 2014;115:68-77.

39. Tancredi V, D'Arcangelo G, Grassi F, Tarroni P, Palmieri G, Santoni A, Eusebi F. Tumor necrosis factor alters synaptic transmission in rat hippocampal slices. Neurosci Lett. 1992;146(2):176-8.

40. Park KM, Bowers WJ. Tumor necrosis factor-alpha mediated signaling in neuronal homeostasis and dysfunction. Cell Signal. 2010;22(7):977-83.

\section{Figures}




\section{Fig 1.}

A

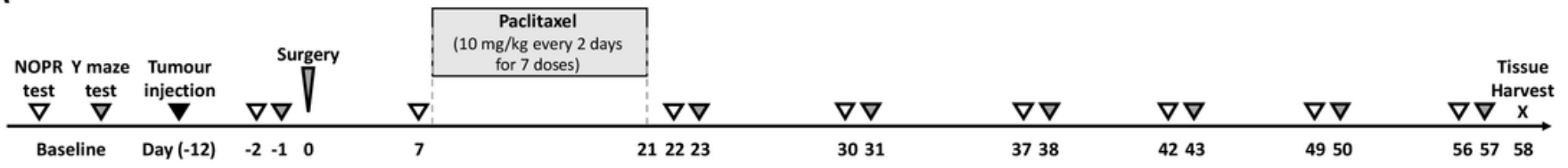

B
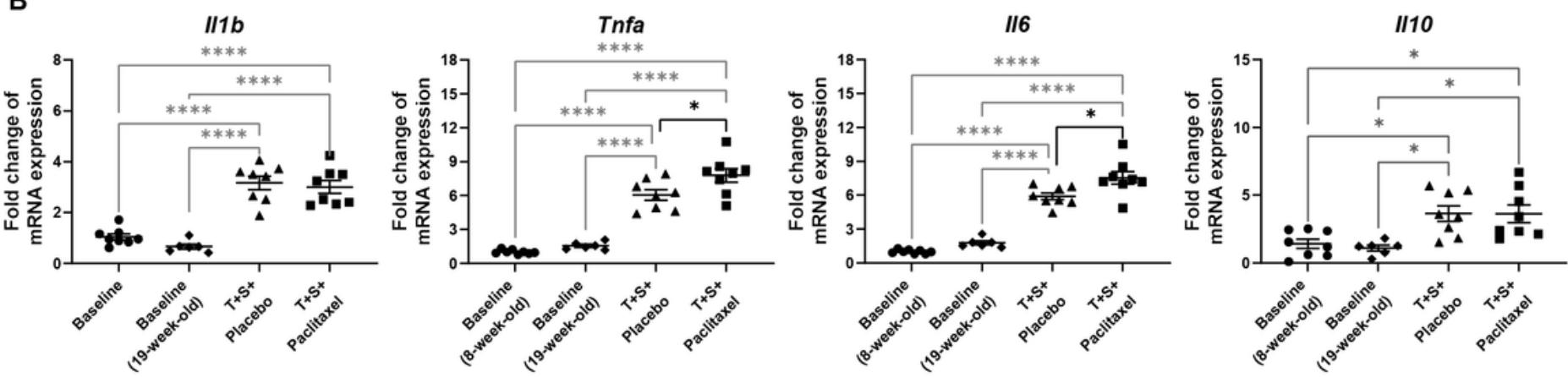

C

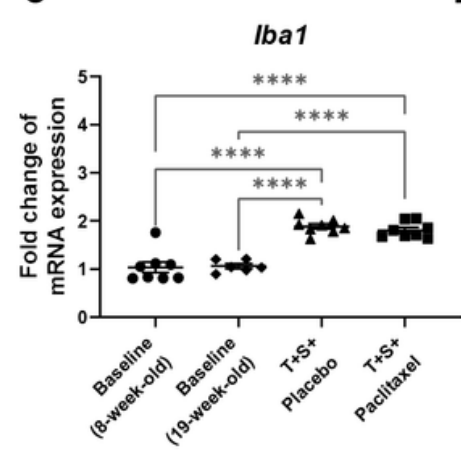

D

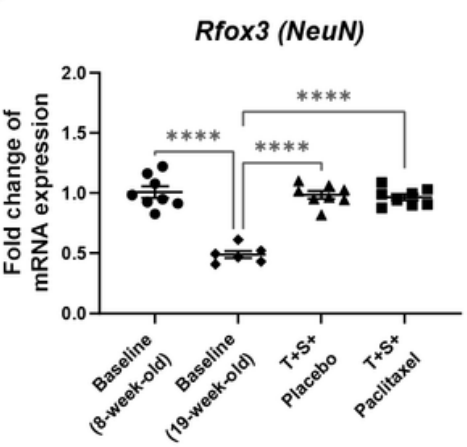

E

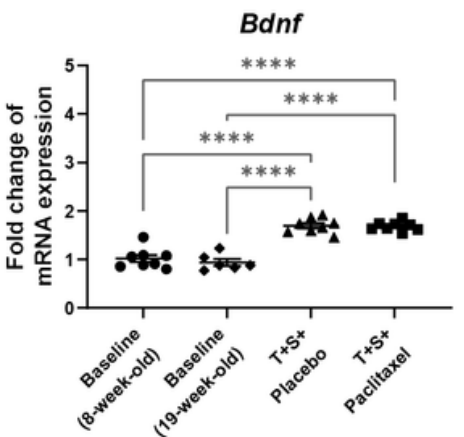

Spleen mass

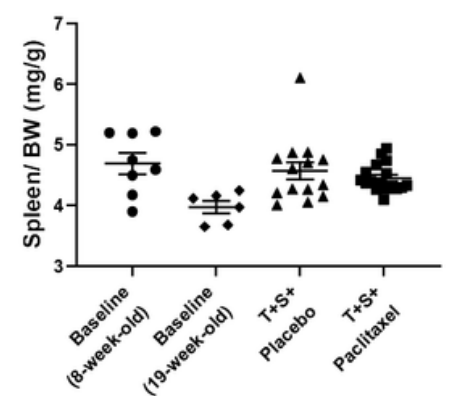

\section{Figure 1}

Paclitaxel exacerbated cancer- and cancer surgery-induced Tnfa and II6 gene expression in the hippocampus (A) Experimental timeline. (B) II1b, Tnfa, Il6, Il10, (C) Iba1, (D) Rfox3 (NeuN), and (E) Bdnf mRNA expression levels in the hippocampus and $(F)$ Spleen mass normalized to body weight (BW) of untreated 8-week-old baseline mice $(n=8)$, untreated 19-week-old baseline mice $(n=6)$, and paclitaxel $(n$ $=8)$ or placebo $(n=8)$ treated mice injected with 67NR tumours that underwent primary tumour resection. Data shown as mean + SE. Comparison between groups is shown as $* p \leq 0.05$ and ${ }^{\star \star \star \star} p \leq 0.0001$ from one-way ANOVA followed by Tukey's multiple comparisons test. $T+S=$ tumours + surgery/ 


\section{Fig 2.}

A

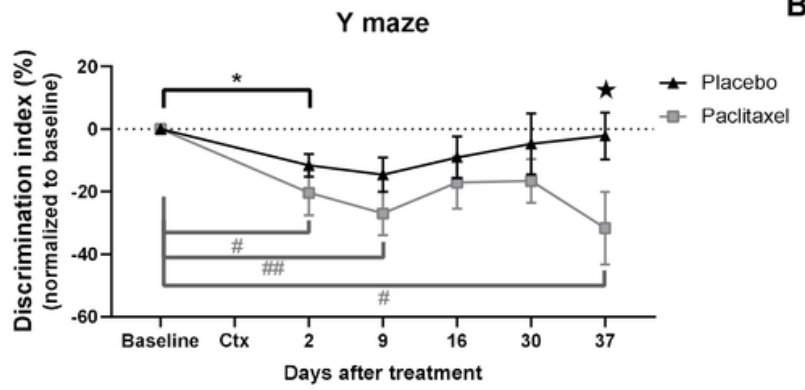

B

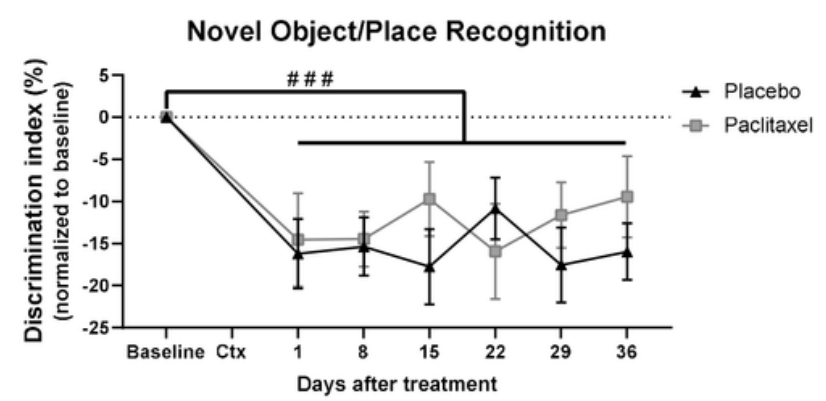

Figure 2

Sustained cognitive impairment induced by cancer, cancer surgery, and chemotherapy (A) Time spent exploring the novel arm in the spatial Y maze test for paclitaxel $(n=16)$ or placebo $(n=10)$ treated mice injected with 67NR tumours that underwent primary tumour resection (B) Time spent exploring the novel object in the NOPR maze test for paclitaxel $(n=15)$ or placebo $(n=13)$ treated mice injected with 67 NR tumours that underwent primary tumour resection. In (A and B), Ctx = the day placebo/paclitaxel injection. Data are represented as mean $+\mathrm{SE}$. Within group comparisons between baseline and other time points is shown as \# $\mathrm{p} \leq 0.05$ using Dunnett's multiple comparison test (A). Main effect of time between baseline and other time points is shown as \#\#\# $p \leq 0.01$ using repeated measures ANOVA (B). Between group comparisons shown as * $p \leq 0.05$ using independent student t-tests. 


\section{Fig 3.}

A

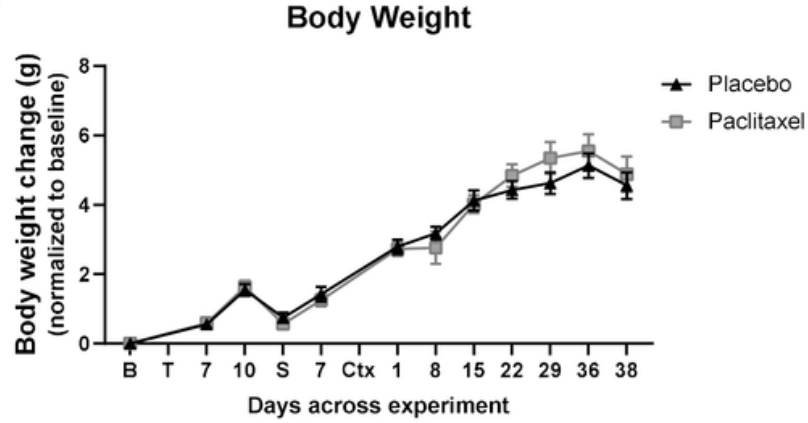

C

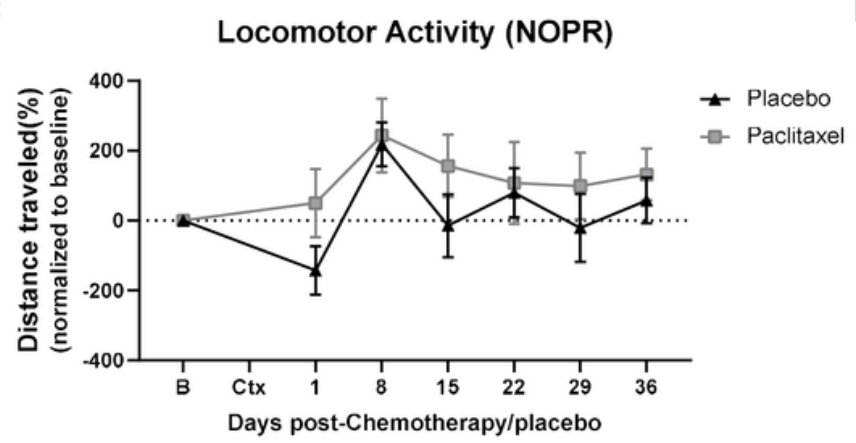

B

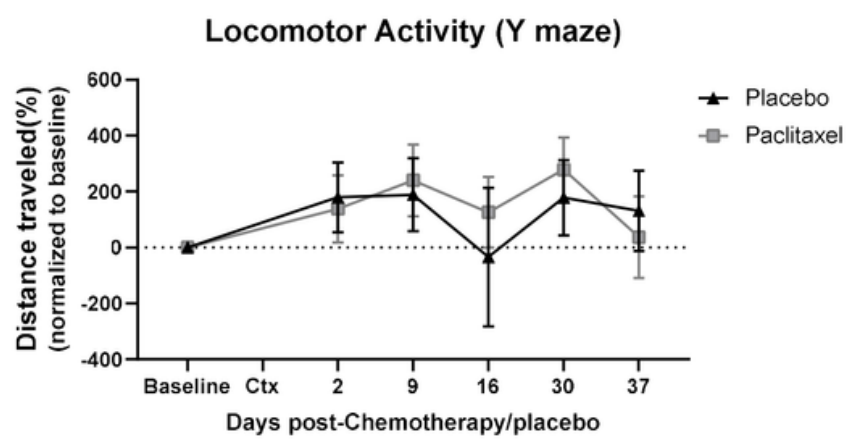

D

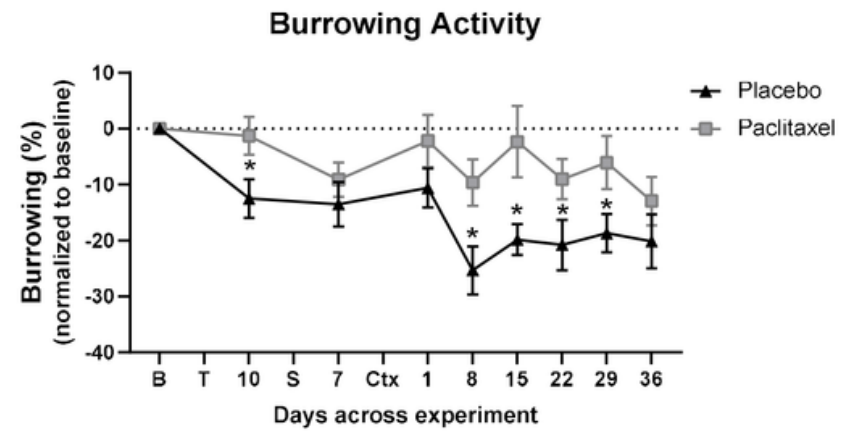

Figure 3

Memory impairment was independent to sickness responses and behaviour. (A) Body weight change (B) Distance traveled in the Y maze test. (C) Distance traveled in the NOPR test. (D) Burrowing activity. In (A$D), B=$ baseline; $T=$ the day of tumour cell injection; $S=$ the day of primary tumour resection; $C t x=$ the day placebo/paclitaxel injection. Mice were injected with 67NR tumours and underwent primary tumour resection before treatment with either paclitaxel $(n=13)$ or placebo $(n=13)$. Data shown as mean $+S E$. Between group comparisons revealed no significant differences between paclitaxel and placebo treated groups but both groups significantly increased in body weight over time $(p<0.0001)(A)$, and in NOPR test locomotor activity $(p<0.001)(C)$. Burrowing activity declined in both groups over the course of the experiment $(p<0.001)(D)$.

\section{Supplementary Files}

This is a list of supplementary files associated with this preprint. Click to download.

- s1.png

- SUPPLEMENTARYMATERIAL.docx 